\title{
FESTIVAL: Towards an intercontinental federation approach
}

Juan R. Santana ${ }^{1}$, José A. Galache ${ }^{1}$, Toyokazu Akiyama ${ }^{2}$, Levent Gurgen ${ }^{3}$, Morito Matsuoka ${ }^{4}$, Martino Maggio ${ }^{5}$ and Shuuichirou Murata ${ }^{6}$

${ }^{1}$ Universidad de Cantabria, Santander, Spain

\{jrsantana, jgalache\} etlmat. unican.es

${ }^{2}$ Kyoto Sangyo University, Kyoto, Japan,

akiyamalcc.kyoto-su.ac.jp

${ }^{3}$ Commissariat à l'énergie atomique et aux énergies alternatives, Grenoble, France,

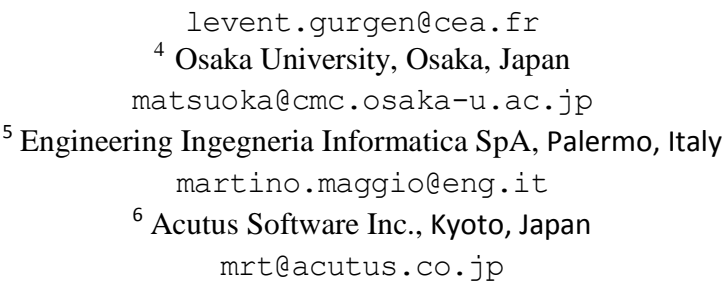

\begin{abstract}
In the last years, in both Europe and Japan, several initiatives have been started with the aim of building and testing Internet of Things and Smart ICT architectures and platforms to address specific domain issues through designed solutions. FESTIVAL EU-Japan collaborative project aims at federating these testbeds, making them interoperable, allowing centralized data collection and analyzing societal issues in both cultures, all of it under a user privacypreserving context. In this sense, FESTIVAL pursues a twofold approach: firstly, the intercontinental federation of testbeds in Japan and Europe using existing tools as well as developing new ones; and secondly, the creation of new services and experiments, to be performed on top of the FESTIVAL testbeds and experimentation facilities, associated to three different smart city domains: smart energy, smart building and smart shopping. Throughout this article the current status of the project (in its first year) is shown, describing the Experimentation as a Service federation approach to be implemented, with a first analysis of the platforms and testbeds that are included within the project. Furthermore, the paper also describes the services and use cases that will be conducted within FESTIVAL lifespan. Finally, next steps to be carried out in the coming years of the project are indicated.
\end{abstract}

Keywords: federation - testbed $\cdot$ platform $\cdot$ experimentation facility $\cdot$ EaaS smart shopping $\cdot$ smart energy $\cdot$ smart building. 


\section{Introduction}

The Internet of Things (IoT) is an emerging paradigm that is radically changing the way we interact with daily life objects at various environments such as home, work, transportation and city [1]. By connecting the physical environment to the internet, it provides real-time information about the environment as well as possibility of controlling it with unexampled easiness. Having access to this cyber-physical environment by just clicking on the button of a smart phone is one step forward to human's dream of controlling everything.

IoT bridges the gap between the cyber and the physical worlds; therefore testbeds allowing verification of IoT solutions must be closely coupled with the physical environments where the experiments will be running. This is an important differentiation point with respect to traditional experimentation testbeds where the virtual resources can be everywhere on the planet and be shared among different experimenters. Real-life testbeds are thus necessary for validating Smart ICT services relying on IoT infrastructures. The experimenters have to be able to gather real-life environmental data, to have direct control to the environment and to interact with the end-users, as main actors for validating these ICT services.

However, IoT testbeds are not only expensive to setup due to the costs of experimentation material (sensors, actuators, communication infrastructure, etc.) but also difficult to deploy, setup and maintain in the physical world. For those stakeholders (researchers, SMEs, application developers, etc.), who do not have such opportunity of deployment, testbeds are crucial and the only way to verify their developments. Besides, if the developments cover several domains requiring different types of physical environments and/or user types, federation of those testbeds becomes the key concept for allowing the access to such valuable resources.

FESTIVAL [2] project's vision is to offer Internet of Things experimentation platforms providing an interaction facility with physical environments and endusers, where experimenters can validate their Smart ICT service developments in various domains. FESTIVAL testbeds will connect cyber world to the physical world, from large scale deployments at a city scale, to small platforms in lab environments, also including dedicated physical spaces simulating real-life settings. Those platforms will be connected and federated via homogeneous access APIs with an "Experimentation as a Service" (EaaS) model for experimenters to test their added value services.

There have been long years of research work in Europe and Japan on federation of testbeds and, more recently, on IoT testbeds. FESTIVAL will reuse as much as possible existing software and hardware components from Europe and Japan for building the EaaS federation. In the European side, several hardware, software and infrastructure enablers from various European Future Internet initiatives such as FIRE, FI-PPP and IERC could be reused, whilst Japanese side will offer hardware and software components from NICT's Smart ICT testbeds.

The paper will show the status of the FESTIVAL project within its first year of development, being structured as follows. In Section II, it is presented the novel EaaS federation approach to be addressed by the FESTIVAL project, thus 
describing in Section III the different experimentation facilities, platforms, living labs and testbeds considered within the project to be federated under the aforementioned EaaS model. Section IV will present the different use cases to be developed within the project scope, indicating the testbed(s) on top of which they will be running. Finally, section $\mathrm{V}$ will indicate some conclusions derived from the work carried out, also indicating the next steps to be achieved in the next years of the project.

\section{EaaS model for experimenters: A federation approach}

As indicated in the introduction section of this document, one of the main objectives of the Festival project is to federate a highly heterogeneous set of facilities from Europe and Japan. For many years, over the FP7 program, the European FIRE initiative made an effort to move from individual or locally clustered future internet testbeds toward actual federation of testbeds. During these years, the federation technologies have matured enough to include a wide variety of tools such as resource discovery and provisioning, resource reservation, experiment control, resource and experiment monitoring, etc. FESTIVAL aims at going one step further by defining and applying the Experimentation as a Service approach. This concept is to be understood as the capability for a federated testbed to provide reconfigurable on-demand access to a set of virtual reusable resources allowing the set-up and running of traceable and reproducible experiments. The creation of this federation platform allows experimenters to rapidly deploy experiments based on new ICT services belonging to different smart city domains, such as smart shopping, smart energy, smart building, or participatory sensing.

The federation within FESTIVAL will take advantage of existing hardware and software components from Europe and in Japan, with the goal of creating APIs that will allow homogenous access to all existing resources in FESTIVAL. In order to guarantee the success in achieving these results, FESTIVAL federation follows a strict roadmap:

- Identify: this step identifies requirements for IoT experimentation testbeds, as well as the existing potential reusable assets in EU and JP ecosystems. The assets can be of different nature such as infrastructures, HW/software platforms, HW/SW components, etc.

- Analyze: once the candidate reusable assets have been identified, a deep analysis into the details to understand possibilities and limitations of the platforms will be carried out. Therefore, besides robust technical components, this part includes the documentation analysis to better understanding of the selected assets.

- Select: After analyzing the assets, a selection of those that are of interest to the project will be performed, also considering the requirements that will be identified at the beginning of the project and the results of the analysis performed in the preceding step. 
- Integrate: The selected useful reusable components will be integrated into the existing target testbeds of the project, where the heterogeneity is a reality as consequence of technical, technological, operational and political specific constraints of each testbed. FESTIVAL will rely on an adaptation approach to make heterogeneous components work together.

- Federate: The testbeds enhanced with the selected assets will be federated by adopting an Experimentation Service Model. The EaaS platform will be based on commonly agreed standards and data models, in order to provide a homogeneous abstraction layer on top of the heterogeneous testbeds.

At the time being, FESTIVAL is finishing the second step in the described roadmap. Within this step, a wide set of interfaces, data formats, middleware entities and access policies have been identified. Furthermore, the analysis also considers other existing federation approaches addressed in other projects such as TEFIS [3] making available a set of testbeds, the TEFIS Testbed Federation, and offering a single access point to this federation for communities of experimenters, FED4FIRE [4] whose goal is to provide a homogenous access to the facilities built under the framework of the FIRE initiative, and FELIX [5], which is an EU-Japan collaborative project based on network infrastructure federation.

The three federation projects mentioned above are based in the existing Slicebased Facility Architecture (SFA) [6], in order to reach the maximum compatibility with other testbeds that use such architecture. The main component used with this federation philosophy, the GENI Aggregate Manager [7], is in charge of the discovery, reservation and provisioning of testbed resources, using their own format for describing the resources, namely RSpec. In spite of the fact that SFA is mainly intended for the management of fully controllable resources (e.g. ssh capable devices), IoT testbeds involved in FED4FIRE, such as SmartSantander [8] and [9], are already implementing these tools as a wrapper of the native testbed tools for IoT device monitoring [10].

On the contrary, the FIWARE [11] initiative can be considered as a fourth federation approach, which has no link with SFA and is also more friendly with IoT-based testbeds. The use of FIWARE for IoT federation can be done through the available GEs (Generic Enablers), which allow fast deployment of different tools for managing IoT devices, as well as the storage of data retrieved by them.

\section{Analysis and classification of FESTIVAL assets}

This section aims at describing European and Japanese assets offered by the different partners within the FESTIVAL project, and will serve as basis for the identification of the best suited strategies for the federation goal.

It is important to make a clear differentiation between platforms, intended as the architectural and software elements that allow the management and use of the deployments; and testbeds, considered as the deployments and infrastructures that are available for external experimenters through a software platform. Apart from 
these groups, two additional ones should be also differentiate: the experimentation facilities, as the physical deployments and available areas for experimentation that can be used to experiment and retrieve data, but that are not still managed by a platform opened for external experimenters; and the living labs, oriented towards user-centric experimentation, involving the users in both the creation and the experimental processes.

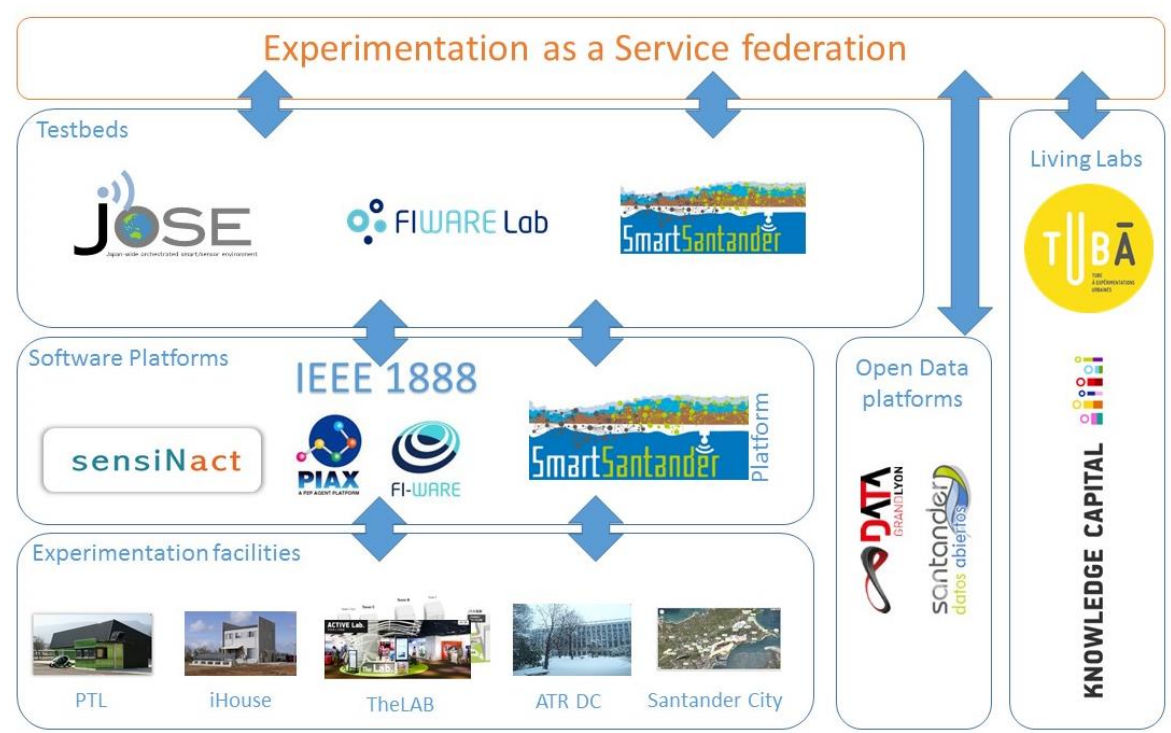

Fig. 1. Relationship between testbeds, platforms, experimentation facilities and living labs

Fig. 1. shows the relationship among the different assets of FESTIVAL, all of them under the federation umbrella that will provide a homogeneous and uniform access.

\subsection{Experimentation facilities}

As previously commented, experimentation facilities are conceived as playgrounds for experimentation, thus offering physical IoT deployments, including the possibility of adding new devices. The main difference between these experimentation facilities and the testbeds consists in the fact that the testbeds also include a software platform accessible from external experimenters to make use of the associated deployments. There are several experimentation facilities identified within FESTIVAL:

- The PTL [12], or the Connectivity Technologies Platform, is a facility to speed up the development of innovative products in emerging strategic fields of Health, Housing and Transport. The PTL is divided in three platforms: the "Connected Home" platform which is intended to evaluate interoperability of heterogeneous systems inside buildings; the "Connected Transport" platform to address 
mobility scenarios to improve safety and mobility connectivity in outdoor areas; and the "Health at Home" platform to retrieve and merge information from environmental, physiological and activity sensors. Higher-level information is transmitted through available communication networks to improve safety, comfort and autonomy.

- The iHOUSE [13] facility is an experimental smart house built by ISICO (Ishikawa Sunrise Industries Creation Organization). The iHouse facility is composed by multiple sensors, such as presence detectors, temperature, humidity, luminosity, door opening/closing sensors, energy meters, wind speed/direction and rain detectors. These sensors can be accessed by ECHONET protocol, that is the communication protocol for EMS (Energy Management Systems), used mainly in Japan.

- The ATR DC is an experimental datacenter facility on the research project funded by MOE (Ministry of Environment) of Japan. The goal of this research facility is to reduce total energy consumption of small-to-middle scale datacenters by up to $70 \%$, by means of various novel ideas on power supply and airflow technologies, task assignment on servers, temperature prediction, and integrated control mechanism. The infrastructure of the facility is comprised of multiple sensors connected to a data center of around 400 servers with 8 racks. Each sensor can access data such as CPU and memory utilization, fan speed or CPU temperatures. In addition, data from around 100 sensors to measure the working temperature of the servers and the information of the infrastructure power consumption are also available.

- Santander City can be considered as an open experimentation space to be used. The city is the facility where the SmartSantander testbed deployment has been carried out. The specific details on this experimentation facility can be found in section 3.3.

- The Lab, explained in detail in section 3.4 , can be also considered as an experimentation facility, thus offering its showrooms for deploying devices, including sensors, to perform the experimentation

\subsection{Software Platforms}

There have been identified two groups of software platforms in FESTIVAL. On the one hand, the platforms that can be used to manage subjacent resources, such as virtual machines or IoT devices, thus supporting the control and management of the resources, including resource description, reservation or data monitoring. On the other hand, they are considered the platforms more oriented towards sharing information through open data policies.

In the first group, five platforms have been identified as candidates to be federated:

- The sensiNact Gateway [14] allows the interconnection of different networks with heterogeneous data formats and protocols, thus enabling the access and 
communication with embedded devices. It is composed of five functional groups and their relative interfaces: i) the device protocol adapter, which allows abstracting the specific connectivity technology of wireless sensor networks; ii) the Smart Object Access and Control, which implements core functionalities such as resource discovering and secure communications; iii) the Consumer API, a protocol-agnostic API to expose services; iv) the Consumer API Protocol Adapter, consisting of a set of bridges to translate the consumer API interface into specific application protocols; v) the Gateway Management functional group, including all the components needed to ease the management of the devices connected to the sensiNact platform; and finally, vi) the Manager Protocol Adapter, which allows adapting the Gateway Management API to the specific protocols used by different external management entities.

- The FIWARE platform, which provides enhanced OpenStack-based cloud hosting capabilities plus a rich library of components including a number of added-value functions offered "as a Service". These components, called Generic Enablers (GEs), provide open standard APIs that make it easier to connect to the Internet of Things, process data and media in real-time at large scale, perform BigData analysis or incorporate advanced features for interaction with the user. Availability of open source Generic Enabler implementations (GEis) will accelerate availability of multiple commercial FIWARE providers, all supporting the same set of APIs. Of particular interest for the EaaS platform developed in FESTIVAL are two of the available GEs: the ORION context broker, an implementation of a Publish/Subscribe broker which allows registering context producer applications, and to subscribe and query context information; and the COSMOS big data, that allows the long-term storage of context information to be available later.

- The SmartSantander platform is the upper layer from the SmartSantander testbed. It is in charge of managing and control deployed resources in the city of Santander as well as external services that might use the platform to store and retrieve data. The SmartSantander platform provides a REST interface to register new producers and, once authenticated, inject data into the platform. It also exposes an interface for external experimenters and service providers so as to access real-time and historical data from SmartSantander. Both interfaces are provided by the IoT API component, which was created to support the federation within FED4Fire. Additionally, the platform also provides the functionality to manage the sensors and services registered in SmartSantander, making use of two components: the resource directory and resource register manager. Finally, the platform also provides the integration with FIWARE, allowing to inject the sensor data into the corresponding instances of the ORION and COSMOS GEs previously explained.

- The PIAX [15] (P2P Interactive Agent eXtensions) is an open source framework that integrates P2P structured overlay network and agent platform. PIAX is implemented by Java. PIAX can be used as a messaging platform for data 
gathering from the sensor network, parallel/distributed data analysis and delivering the analysis results to the actuators for facility controllers and visualization devices like digital signage. PIAX has its own testbed operated by NICT and it can be used to test PIAX Agent implementation easily.

- The IEEE1888 [16] is a standardized facility information access protocol (FIAP). IEEE1888 has a reference implementation of its communication protocol and data storage with standardized data format. Along with JOSE, it provides a platform which extends the IEEE1888 reference implementation to fit a large scale distributed computing environment. The extended implementation includes overlay routing function among storage instances of IEEE1888 (FIAP Storage) by using PIAX platform.

Regarding to the second group, the platforms more oriented towards sharing information through open data policies, next ones can be indicated:

- The Metropole of Lyon's Open Data [17] is a pro-innovation platform based on the Open Data paradigm. By providing wide-ranging access to public data such as the land register map for the conurbation, the surface area taken up by greenery, the availability of shared bikes (Vélo'v) or the locations of automatic car-sharing stations, the Greater Lyon SmartData platform is addressing the issues of data accessibility, which have been made objectives of the smart city. It includes a new series of data principally for the mobility sector, such as real-time traffic, highway events, traffic history or the number of available bicycles in Lyon.

- The Santander Open Data [18] is a platform deployed by the Santander City Council, offering official and public data in exploitable formats to the citizens, so they can reuse it for their own purposes, such as development of new services or the provision of added value to the existing ones. Among the data publicly offered, those related with transport, urban planning and infrastructure, shops, demography, society and well-being, culture and leisure events, are the most representative ones.

\subsection{Testbeds}

The testbeds within FESTIVAL are basically composed of a resource deployment, like the experimentation facilities explained above, and a software platform that supports the experimentation on top of them, allowing external users to perform their experiments, either deploying new software or making use of the data being produced in the testbed.

This section details the testbeds that belong to FESTIVAL and will be finally federated to be part of the EaaS platform. Among the testbeds we can identified two types: the Infrastructure provider testbeds that are the group of testbeds that 
provide infrastructure resources, such as virtual machines or SDN-based Service Orchestration, to perform experiments; and the IoT infrastructure testbeds.

Regarding the first type of testbeds, two can be indicated:

- The JOSE testbed [19], a Japan-wide open testbed which consists of a large number of wireless sensors, SDN capabilities and distributed "Cloud" resources along Japan. The facilities of JOSE are connected via high-speed network with SDN feature, aiming at accelerating field trials of "large-scale smart ICT services", essential for building future smart societies. JOSE has the following main characteristics: a huge amount of computation resources, a dedicated "sensor network" provision with SDN capabilities and a "takeout" sensor facilities for users' own experiments where many field trials can coexist.

- The Engineering FIWARE-Lab [20], stands as an instance of FI-WARE based on a cloud environment that allows the users to deploy, configure and execute a set of Generic Enablers. The cloud infrastructure is based on OpenStack, an open source software for creating cloud platforms and is directly managed by Engineering, which will provide specific computational resources dedicated to the FESTIVAL project. The FIWARE-Lab provides the possibility to create virtual machines and resources to deploy and execute the FIWARE Generic Enablers, with an existing set of preconfigured virtual images.

Regarding to the IoT infrastructure testbeds: they are composed of all the testbeds that offer a set of IoT devices that can be used to perform experiments either using their own sensor data or the data from deployed experiments in each device. In the case of FESTIVAL, the SmartSantander testbed provides an experimentation test facility for the research and experimentation of architectures, key enabling technologies, services and applications for the Internet of Things in the context of a city. The SmartSantander is not just limited to the already deployed devices but it is flexible enough to add new ones.

\subsection{Living Labs}

As previously explained, this last category refers to those facilities where the user stands as the key issue of the experimentation, considering not only the useful data that the user can provide, e.g. giving feedback about a new prototype, but also as part of the co-creation process, making them participants of the actual research. The next two living labs are part of FESTIVAL and will be also part of the federation goal:

- Lyon Urban Data is an association based on a mixed consortium of public and private entities that operates a place named TUBA [21], which stands for "Experimentation Urban Test Tube". The TUBA is a $600 \mathrm{~m}^{2}$ place dedicated to experimenting on new services and helping developing new projects (from startup, SMEs and large companies), based on available data. It is mainly composed of two spaces: The LAB, a 180 square meters showroom fully opened 
to the citizen, where they are encourage to contribute with their own ideas and feedback about what is being shown; and The Mix, a 420 square meters dedicated to show the last innovations advances from the TUBA partners as well as other public or private entities involved in the Smart City innovation.

- The Lab. [22] is the core facility in Knowledge Capital, an innovation space placed in the Grand Front Osaka building. The Lab. is a showcase where general public, such as researchers, creators, artists, students, senior citizens, housewives and children, can experience the latest technologies and have interactions with other exhibitors. The Lab. constitutes a space that attracts global prototypes and world-leading technologies, and is a hub from which the latest strains of culture emanate. Visitors not only get to see and touch ingenious inventions, but are also given the chance to participate in the creative process, fitting the description of this space as a laboratory.

\section{$4 \quad$ Festival use cases}

In order to exploit the potential of each of the testbeds as well as to take advantage of the additional features offered by the EaaS federation, several use cases and ICT services have been defined, mainly associated to three domains within the smart city context: energy, building and shopping. Use cases are framed in two categories: the application ones as those addressing services for end users under a real environment; and the experimentation use cases, intended to specifically study the behavior of current and cutting-edge technologies over an outdoor testbed.

Apart from the aforementioned use cases are mainly intended on the one hand to be applied in specific testbeds or platforms for improving or generating new services such the use of cameras for different applications within the scope of smart building; and on the other hand, comparing behaviors associated to different testbeds and cities, such as those indicated for the smart shopping; others will be also described covering the use of the EasS model previously shown, thus taking advantage offered by the federation of several testbeds.

\subsection{Smart Energy}

Regarding to the application use cases, energy monitoring in buildings on top of PTL platform for metering consumed resources (water, gas, electricity), thus adapting the different parameters to the usage and presence in buildings, as well as study of energy management systems (xEMS) over different experimentation spaces such as The Lab, ATR DC and iHouse, in order to realize large-scale application service providers for various existing local xEMSs: HEMS (Home), FEMS (Factory), DEMS (Datacenter), CEMS (Community); and to contribute to 
global optimization of power consumption and overhead reduction of local EMSs. Apart from this application use case, regarding to the study of xEMS, two experimentation use cases are also planned on top of JOSE testbed and PIAX platform, thus allowing the experimenter to reserve several resources (sensors and $\mathrm{VMs}$ ) according to an utilization plan sent to the administrator, connecting his own sensors and actuators and receiving the corresponding measurements retrieved by them.

\subsection{Smart Building}

From the smart building perspective, different use cases based on the use of smart cameras will be carried out thus considering on the one hand, the use of untraditional cameras that allows extracting some useful features of filmed scenes without the acquisition of "real images" and, on the other hand the use of traditional image sensors with dedicated image processing for extracting image features without storing image flows, over PTL space as well as TUBA and The Lab testbeds. The use of these cameras will be applied to queue management for counting people in a queue or a determined area (over TUBA and PTL), to adaptability issues in controlling media such as sounds, displayed videos and advertising (over TUBA) and to human- human interaction, thus capturing and streaming video in real-time for visually "connecting" people from separate places (over TUBA and The Lab).

\subsection{Smart Shopping}

Use cases on Smart Shopping are related with gathering different measurements, both environmental, presence detection and user tracking, within shops environment, thus accordingly processing the received data and offering contextaware incentives (e.g. specific discounts) to the users, all of them over the SmartSantander testbed. In the same direction, over TheLab testbed pedestrian flows will be captured, thus proceeding to recommend certain shops to the users according to their degree of crowdedness, as well as changing the background music, light, aroma for making shopping experience more pleasant to the user.

\subsection{EaaS specific use cases}

Regarding to EaaS model, it is described the use of IoT-based experimentation, mainly based on SmartSantander testbed, thus reserving and accessing to the different IoT devices by sending/receiving commands or flashing them in order to 
run a specific experiment. For both accessing to the data and processing them accordingly to generate an application, the experimenter can access to the virtual machine resources offer within the Festival federated platform. Additionally to this use case, another interesting one consists on using FIWARE-Lab generic enablers (GEs), so that the experimenter specifies the objectives of the experiment, the duration, the needed computational resources and the GE that she wants to use. Once decided, the images of the GEs will be installed in the different virtual machines offered within the federated Festival platform.

\section{Conclusions and future steps}

As the technologies of the Future Internet move ever closer to the market, in an ever shorter innovation cycle, the need to validate the experimentation in "real life" trials with end user is becoming crucial. In this sense, the development of an open federation of testbeds enabled by the EaaS model, posed within the FESTIVAL project, will have a real impact on the number and quality of the experimentations that are run on the testbed(s), thus presenting both small and large scale trials over various application domains.

In order to achieved and assess (within the project lifetime) the aforementioned EaaS model, a set of testbeds, as well as platforms, living labs and experimentation facilities, have been described detailing their main characteristics and available resources, as the first step towards the federation of them under this EaaS model. Additionally, in order to show the potential of these testbeds, as enablers for real smart city applications, they have been presented the use cases to be carried out during the lifetime of the project, mainly associated to three main pillars: smart energy, smart building and smart shopping.

Finally, and considering that the FESTIVAL project is still in its first year of progress, the definition and implementation of a whole architecture (basis of the EaaS model) for accessing and managing of the testbeds in a homogenized and uniform way (common interfaces, APIs, access protocols, etc.), will be one of the main objectives in the second year of the project. Additionally, the translation of testbed-related use cases into commercial ICT services on top of the aforementioned architecture, will pose as another important challenge to be achieved.

Acknowledgments. This work was funded in part by the European Union's Horizon 2020 Programme of the FESTIVAL project (Federated Interoperable Smart ICT Services Development and Testing Platforms) under grant agreement 643275, and from the Japanese National Institute of Information and Communications Technology. 


\section{References}

1. Botterman, M., Internet of things: an early reality of the future internet, in: Workshop Report prepared for European Commission, Information Society and Media Directorate General, Networked Enterprise \& RFID Unit (D4), Prague (2009)

2. FESTIVAL project, http://www.festival-project.eu/

3. TEFIS project, http://www.ict-fire.eu/home/success-stories/tefis.html

4. FED4Fire project, http://fed4fire.eu/

5. FELIX project, http://www.ict-felix.eu/

6. Peterson, L., Ricci, R., Falk, A., Chase, J., Slice-based federation architecture (sfa). Working draft, Version 2.0 (2010)

7. GENI AM API, http://groups.geni.net/geni/wiki/GAPI_AM_API_V3

8. SmartSantander project, http://smartsantander.eu/

9. Sanchez, L., Muñoz, L., Galache, J.A., Sotres, P., Santana, J.R., Gutierrez, V., Ramdhany, R., Gluhak, A., Krco, S., Theodoridis, E., Pfisterer, D., "Smartsantander: IoT Experimentation over a Smart City Testbed", Computer Networks J., vol. 61, pp.217-238 (2014)

10.FED4Fire D4.5 - Report on second cycle developments of the services and applications community

11.FIWARE initiative, https://www.fiware.org/

12.PTL testbed, http:/www.leti.fr/fr/layout/set/print/layout/set/print/content/download/2467/29637/file/3 - SESSION B3 - Laurent-Fr\%C3\%A9d\%C3\%A9ric Ducreux.pdf

13. iHouse testbed, http://www.echonet.gr.jp/echo/example/pdf/example06.pdf

14. BUTLER Project, http://www.iot-butler.eu/

15. PIAX platform, https://piax.jgn-x.jp/pat/help/en/aboutPat/aboutPat.html

16. IEEE1888 reference implementation, http://fiap-develop.gutp.ic.i.u-tokyo.ac.jp/dist/

17. Metropole of Lyon's Open Data, http://data.grandlyon.com/

18. Santander Open Data, http://datos.santander.es/

19. Teranishi. Y., "JOSE: Japan-wide orchestrated smart ICT testbed for future smart society", http://cordis.europa.eu/fp7/ict/future-networks/documents/eu-japan/stream-cteranishi.pdf

20. FIWARE LAB, https://fiware.eng.it

21. TUBA living lab, http://www.tuba-lyon.com/

22. theLab. Knowledge Capital, https://kc-i.jp/en/ 